\title{
Complex Convexity of Orlicz Modular Sequence Spaces
}

\author{
Lili Chen, ${ }^{1,2}$ Deyun Chen, ${ }^{1}$ and Yang Jiang ${ }^{2}$ \\ ${ }^{1}$ Post-Doctoral Station for Computer Science and Technology, Harbin University of Science and Technology, Harbin 150080, China \\ ${ }^{2}$ Department of Mathematics, Harbin University of Science and Technology, Harbin 150080, China
}

Correspondence should be addressed to Lili Chen; cll2119@hotmail.com

Received 28 July 2016; Accepted 12 October 2016

Academic Editor: Richard I. Avery

Copyright (C) 2016 Lili Chen et al. This is an open access article distributed under the Creative Commons Attribution License, which permits unrestricted use, distribution, and reproduction in any medium, provided the original work is properly cited.

\begin{abstract}
The concepts of complex extreme points, complex strongly extreme points, complex strict convexity, and complex midpoint locally uniform convexity in general modular spaces are introduced. Then we prove that, for any Orlicz modular sequence space $l_{\Phi, \rho}, l_{\Phi, \rho}$ is complex midpoint locally uniformly convex. As a corollary, $l_{\Phi, \rho}$ is also complex strictly convex.
\end{abstract}

\section{Introduction}

In 1967, the notions of complex extreme points and complex strict convexity have been introduced by Thorp and Whitley [1]. They proved that the strong maximum modulus theorem for analytic functions with values in a complex Banach space $X$ holds true whenever each point of the unit sphere of $X$ is a complex extreme point. In 1975, Globevnik further introduced the notions of complex strict and uniform convexity of complex normed spaces and proved that the complex space $L_{1}$ is complex uniformly convex (see [2]). Davis et al. in [3] investigated the complex convexity of quasi-normed linear spaces. Dowling et al. in [4] studied the complex convexity of Lebesgue-Bochner function spaces. Blasco and Pavlović in [5] obtained sufficient and necessary conditions for a complex Banach space $X$ which is $p$-uniformly PL-convex. Choi et al. in [6] obtained criteria for complex extreme points, complex rotundity, and complex uniform convexity in Orlicz-Lorentz spaces. Hudzik and Narloch in [7] considered relationships between monotonicity and complex rotundity; for instance, a point $f$ of the complexification $E^{C}$ of a real Köthe space $E$ is a complex extreme point if and only if $|f|$ is a point of upper monotonicity in $E$. Lee in $[8,9]$ continued to study relationships between monotonicity and complex convexity in Banach lattices and Quasi-Banach lattices, respectively. Czerwińska and Kamińska in [10] discussed the complex rotundity and midpoint local uniform convexity in symmetric spaces of measurable operators. Recently, Czerwińska and Parrish in [11] characterized complex extreme points in Marcinkiewicz spaces.

In this paper, we introduce the concepts of complex extreme points, complex strongly extreme points, complex strict convexity, and complex midpoint locally uniform convexity in general modular spaces. Then we prove that, for any Orlicz modular sequence space $l_{\Phi, \rho}, l_{\Phi, \rho}$ is complex midpoint locally uniformly convex. As a corollary, $l_{\Phi, \rho}$ is also complex strictly convex.

Before starting with our results, we need to recall some basic concepts and facts of the theory of modular spaces and Orlicz spaces.

Let $X$ be a vector space over the complex field $\mathbb{C}$. A functional $\rho: X \rightarrow[0, \infty]$ is called a modular provided that, for any $f, g \in X$,

(a) $\rho(f)=0$ if and only if $f=0$;

(b) $\rho(\alpha f)=\rho(f)$ for any $\alpha \in \mathbb{C}$ with $|\alpha|=1$;

(c) $\rho(\alpha f+\beta g) \leq \rho(f)+\rho(g)$ for any $\alpha, \beta \geq 0$ with $\alpha+\beta=$ 1 ; we can replace (c) by the following;

$\left(c^{\prime}\right) \rho(\alpha f+\beta g) \leq \alpha \rho(f)+\beta \rho(g)$ for any $\alpha, \beta \geq 0$ with $\alpha+\beta=1$; in this case the modular $\rho$ is said to be a convex modular. A modular space $X_{\rho}$ is defined by

$$
X_{\rho}=\{f \in X: \rho(\lambda f) \longrightarrow 0 \text { as } \lambda \longrightarrow 0\} .
$$


Let $X_{\rho}$ be a modular space; then

$$
\begin{aligned}
& B\left(X_{\rho}\right)=\left\{x \in X_{\rho}: \rho(x) \leq 1\right\}, \\
& S\left(X_{\rho}\right)=\left\{x \in X_{\rho}: \rho(x)=1\right\}
\end{aligned}
$$

denote the closed unit ball and the unit sphere of $X_{\rho}$, respectively. In the sequel $\mathbb{N}, \mathbb{R}$, and $\mathbb{C}$ denote the set of natural numbers, the set of real numbers, and the set of complex numbers, respectively. Let $i$ be the complex number satisfying $i^{2}=-1$.

A map $\Phi: R \rightarrow[0, \infty]$ is said to be an Orlicz function if $\Phi$ is vanishing at zero, even, convex, and continuous and satisfies $\lim _{u \rightarrow 0}(\Phi(u) / u)=0$ and $\lim _{u \rightarrow \infty}(\Phi(u) / u)=\infty$. For every Orlicz function $\Phi$, its complementary function $\Psi$ : $R \rightarrow[0, \infty]$ is defined by the formula

$$
\Psi(v)=\sup \{u|v|-\Phi(u): u \geq 0\},
$$

and the complementary function $\Psi$ is also an Orlicz function.

Define

$$
l^{c}=\left\{x=(x(j))_{j=1}^{\infty}: x(j) \in \mathbb{C}, j=1,2, \ldots\right\} .
$$

For any $x \in l^{c}$, let $\operatorname{supp}(x)=\{j \in \mathbb{N}: x(j) \neq 0\}$ denote the support set of $x$. For a given $N$-function $\Phi$, we define on $l^{c}$ a convex modular by

$$
\rho_{\Phi}(x)=\sum_{j=1}^{\infty} \Phi(|x(j)|), \quad \forall x=(x(j))_{j=1}^{\infty} \in l^{c},
$$

which is said to be an Orlicz modular. The modular space induced by an Orlicz modular is said to be the Orlicz sequence space $l_{\Phi}$. Indeed, Orlicz sequence spaces and their kinds of generalizations belong to modular spaces. For the sake of simplicity, we denote $l_{\Phi, \rho}=\left(l_{\Phi}, \rho_{\Phi}\right)$ and $l_{\Phi}=\left(l_{\Phi},\|\cdot\|\right)$.

For more details on Orlicz spaces we refer to [12-24], and for more details on modular spaces one can consult [25-33].

\section{Main Results}

In this section, we always assume that $\rho$ is a convex modular. It is known that extreme points which are connected with strict convexity of the whole spaces are the most basic and important geometric points in geometric theory of spaces. In [1], Thorp and Whitley first introduced the concepts of complex extreme points and complex strict convexity when they studied the conditions under which the strong maximum modulus theorem for analytic functions always holds in a complex Banach space.

Definition 1 (see $[1])$. Let $(X,\|\cdot\|)$ be a Banach space. A point $x \in S(X)$ is said to be a complex extreme point of $B(X)$ if for every nonzero $y \in X$ there holds $\sup _{|\lambda| \leq 1}\|x+\lambda y\|>1$. A Banach space $X$ is said to be complex strictly convex if every element of $S(X)$ is a complex extreme point of $B(X)$.

In [12], we further studied the notions of complex strongly extreme points and complex midpoint locally uniform convexity in general complex spaces. For more details on notions of complex extreme points and complex strongly extreme points we refer to $[12,13]$.

Definition 2 (see $[12])$. Let $(X,\|\cdot\|)$ be a Banach space. A point $x \in S(X)$ is said to be a complex strongly extreme point of $B(X)$ if, for every $\varepsilon>0$, one has $\Delta_{c}(x, \varepsilon)>0$, where

$$
\begin{aligned}
& \Delta_{c}(x, \varepsilon)=\inf \left\{1-|\lambda|: \exists y \in X \text { s.t. }\left\|x \pm \frac{y}{\lambda}\right\|\right. \\
& \left.\leq 1,\left\|x \pm i \frac{y}{\lambda}\right\| \leq 1,\|y\| \geq \varepsilon\right\} .
\end{aligned}
$$

A Banach space $X$ is said to be complex midpoint locally uniformly convex if every element of $S(X)$ is a complex strongly extreme point of $B(X)$.

Now we generalize the notions of complex extreme points and complex strongly extreme points of Banach spaces to the modular spaces.

Definition 3. Let $X_{\rho}$ be a modular space. A point $x \in S\left(X_{\rho}\right)$ is said to be a complex extreme point of $B\left(X_{\rho}\right)$ if, for any $y \in X_{\rho}$ with $y \neq 0$, there holds

$$
\sup _{|\lambda| \leq 1} \rho(x+\lambda y)>1
$$

$X_{\rho}$ is said to be complex strictly convex if every element of $S\left(X_{\rho}\right)$ is a complex extreme point of $B\left(X_{\rho}\right)$.

Definition 4. Let $X_{\rho}$ be a modular space. A point $x \in S\left(X_{\rho}\right)$ is said to be a complex strongly extreme point of $B\left(X_{\rho}\right)$ if $\Delta_{c, \rho}(x, \varepsilon)>0$ for every $\varepsilon>0$, where

$$
\begin{gathered}
\Delta_{c, \rho}(x, \varepsilon)=\inf \left\{1-|\lambda|: \exists y \in X_{\rho} \text { s.t. } \rho\left(x \pm \frac{y}{\lambda}\right)\right. \\
\left.\leq \frac{1}{|\lambda|}, \rho\left(x \pm i \frac{y}{\lambda}\right) \leq \frac{1}{|\lambda|}, \rho(y) \geq \varepsilon\right\}
\end{gathered}
$$

and $\lambda \in \mathbb{C}$ with $0<|\lambda| \leq 1 . X_{\rho}$ is said to be complex midpoint locally uniformly convex if every element of $S\left(X_{\rho}\right)$ is a complex strongly extreme point of $B\left(X_{\rho}\right)$.

Proposition 5. Let $x \in S\left(X_{\rho}\right), y \in X_{\rho}$, and $m \geq 1$; then the following conditions are equivalent:

(i) $\sup _{|\lambda| \leq 1} \rho(x+\lambda y) \leq m$ for all $\lambda \in \mathbb{C}$ with $|\lambda| \leq 1$.

(ii) $\rho(x \pm y) \leq m$ and $\rho(x \pm i y) \leq m$.

Proof. (i) $\Rightarrow$ (ii) is trivial. For any $\lambda \in \mathbb{C}$ with $|\lambda| \leq 1$, without loss of generality, there exist $k_{1}, k_{2} \in[0,1]$ such that

$$
x+\lambda y=k_{1}(x+y)+k_{2}(x+i y) \text {. }
$$

It follows that

$$
\begin{gathered}
k_{1}+k_{2}=1, \\
k_{1}+i k_{2}=\lambda .
\end{gathered}
$$


Consequently,

$$
\begin{aligned}
\rho(x+\lambda y) & =\rho\left(k_{1}(x+y)+k_{2}(x+i y)\right) \\
& \leq k_{1} \rho(x+y)+k_{2} \rho(x+i y) \leq m .
\end{aligned}
$$

Remark 6. By Proposition 5, it is easy to see that the inequality in Definition 3

$$
\sup _{|\lambda| \leq 1} \rho(x+\lambda y) \leq 1
$$

is equivalent to the inequality $\max _{\lambda= \pm 1, \pm i} \rho(x+\lambda y) \leq 1$.

Next, we give a new modulus which is associated with complex strongly extreme points.

Definition 7. Let $X_{\rho}$ be a modular space and $x \in S\left(X_{\rho}\right)$. For each $\varepsilon>0$, one defines a function as follows:

$$
\delta_{c, \rho}(x, \varepsilon)=\inf \left\{\sup _{|\lambda| \leq 1} \rho(x+\lambda y)-1: \rho(y) \geq \varepsilon\right\} .
$$

Observe that $\delta_{c, \rho}(x, \varepsilon) \geq 0$ for all $\varepsilon>0$.

Proposition 8. Let $X_{\rho}$ be a modular space. A point $x \in S\left(X_{\rho}\right)$ is a complex strongly extreme point of $B\left(X_{\rho}\right)$ if and only if $\delta_{c, \rho}(x, \varepsilon)>0$ for all $\varepsilon>0$.

Proof.

Necessity. Let $x \in S\left(X_{\rho}\right)$ be a complex strongly extreme point of $B\left(X_{\rho}\right)$. Assume that there exists $\varepsilon_{0}>0$ such that $\delta_{c, \rho}\left(x, \varepsilon_{0}\right)=0$. By Definition 7 , we can find a sequence $\left\{y_{n}\right\} \subseteq$ $X_{\rho}$ satisfying $\rho\left(y_{n}\right) \geq \varepsilon_{0}$ and

$$
\sup _{|\lambda| \leq 1} \rho\left(x+\lambda y_{n}\right) \longrightarrow 1 \text { as } n \longrightarrow \infty \text {. }
$$

Let $\alpha_{n}=\sup _{|\lambda| \leq 1} \rho\left(x+\lambda y_{n}\right)$ and $\beta_{n}=1 / \alpha_{n}$. By (14) and noticing that $0<\beta_{n} \leq 1$ and $\beta_{n} \rightarrow 1$ as $n \rightarrow \infty$, we obtain

$$
\begin{gathered}
\rho\left(x \pm \beta_{n} y_{n}\right) \leq \frac{1}{\beta_{n}}, \\
\rho\left(x \pm i \beta_{n} y_{n}\right) \leq \frac{1}{\beta_{n}},
\end{gathered}
$$

which implies $\Delta_{c, \rho}\left(x, \varepsilon_{0}\right)=0$, a contradiction.

Sufficiency. Suppose that $x \in S\left(X_{\rho}\right)$ is not a complex strongly extreme point of $B\left(X_{\rho}\right)$ and $\delta_{c, \rho}(x, \varepsilon)>0$ for all $\varepsilon>0$. By Definition 4 , then there exists $\varepsilon_{0}>0$ such that $\Delta_{c, \rho}\left(x, \varepsilon_{0}\right)=0$. Hence, there are $\lambda_{n} \in \mathbb{C}$ with $\left|\lambda_{n}\right| \rightarrow 1$ and $y_{n} \in X_{\rho}$ satisfying $\rho\left(y_{n}\right) \geq \varepsilon_{0}$, such that

$$
\begin{gathered}
\rho\left(x \pm \frac{y_{n}}{\lambda_{n}}\right) \leq \frac{1}{\left|\lambda_{n}\right|} \\
\rho\left(x \pm i \frac{y_{n}}{\lambda_{n}}\right) \leq \frac{1}{\left|\lambda_{n}\right|}
\end{gathered}
$$

for each $n \in \mathbb{N}$. Setting $z_{n}=y_{n} / \lambda_{n}$, we have

$$
\begin{aligned}
\rho\left(z_{n}\right) & =\rho\left(\frac{y_{n}}{\lambda_{n}}\right) \geq \frac{1}{\left|\lambda_{n}\right|} \rho\left(y_{n}\right) \geq \frac{\varepsilon_{0}}{\left|\lambda_{n}\right|} \geq \varepsilon_{0}, \\
\rho\left(x \pm z_{n}\right) & \leq \frac{1}{\left|\lambda_{n}\right|}, \\
\rho\left(x \pm i z_{n}\right) & \leq \frac{1}{\left|\lambda_{n}\right|} .
\end{aligned}
$$

In view of Proposition 5, we deduce that

$$
\sup _{|\lambda| \leq 1} \rho\left(x+\lambda z_{n}\right) \leq \frac{1}{\left|\lambda_{n}\right|}
$$

It follows that

$$
\begin{aligned}
\delta_{c, \rho}\left(x, \varepsilon_{0}\right) \leq \sup _{|\lambda| \leq 1} \rho\left(x+\lambda z_{n}\right)-1 \leq \frac{1}{\left|\lambda_{n}\right|}- & \longrightarrow 0 \\
\text { as } n & \longrightarrow \infty,
\end{aligned}
$$

which contradicts the fact that $\delta_{c, \rho}(x, \varepsilon)>0$ for all $\varepsilon>0$.

In Banach spaces, we have shown that if $x \in S(X)$ is a complex strongly extreme point of $B(X)$, then $x$ is a complex extreme point of $B(X)$ (see [12]). We will prove that it is also true in modular spaces.

Theorem 9. Let $X_{\rho}$ be a modular space. If $x \in S\left(X_{\rho}\right)$ is a complex strongly extreme point of $B\left(X_{\rho}\right)$, then $x$ is a complex extreme point of $B\left(X_{\rho}\right)$.

Proof. Suppose that $x \in S\left(X_{\rho}\right)$ is not a complex extreme point of the closed unit ball $B\left(X_{\rho}\right)$. Then there exists $z \in X_{\rho} \backslash\{0\}$ such that

$$
\sup _{|\lambda| \leq 1} \rho(x+\lambda z) \leq 1 .
$$

Hence, we have

$$
\begin{gathered}
\rho(x \pm z) \leq 1, \\
\rho(x \pm i z) \leq 1 .
\end{gathered}
$$

Letting $\varepsilon_{0}=\rho(z)>0$, then we obtain $\Delta_{c, \rho}\left(x, \varepsilon_{0}\right)=0$ which is a contradiction.

The following result is an immediate corollary of the previous theorem.

Corollary 10. Let $X_{\rho}$ be a modular space. If $X_{\rho}$ is complex midpoint locally uniformly convex, it is also complex strictly convex.

Next, we will study complex convexity of Orlicz modular sequence spaces. Before starting with our results, let us recall a useful lemma. 
Lemma 11 (see [13]). For any $\varepsilon>0$, there exists $\delta \in(0,1 / 2)$ such that if $u, v \in \mathbb{C}$ and

$$
|v| \geq \frac{\varepsilon}{8} \max _{e}|u+e v|
$$

then

$$
|u| \leq \frac{1-2 \delta}{4} \Sigma_{e}|u+e v|
$$

where

$$
\begin{aligned}
& \max _{e}|u+e v| \\
& \quad=\max \{|u+v|,|u-v|,|u+i v|,|u-i v|\}, \\
& \Sigma_{e}|u+e v|=|u+v|+|u-v|+|u+i v|+|u-i v| .
\end{aligned}
$$

In [34], we have given criteria for complex strict convexity and complex midpoint locally uniform convexity of Orlicz sequence spaces equipped with the $p$-Amemiya norm.

Theorem 12 (see [34]). Assume $1 \leq p<\infty$; then the following are equivalent:

(i) $l_{\Phi, p}$ is complex midpoint locally uniformly convex.

(ii) $l_{\Phi, p}$ is complex strictly convex.

(iii) $a_{\Phi}=0$.

Theorem 13 (see [34]). If $p=\infty$, then $l_{\Phi, p}$ is complex strictly convex if and only if
(i) $\Phi \in \Delta_{2}$,
(ii) $a_{\Phi}=0$.

Theorem 14 (see [34]). If $p=\infty$, then $l_{\Phi, p}$ is complex midpoint locally uniformly convex if and only if

(i) $\Phi \in \Delta_{2}$,

(ii) $a_{\Phi}=0$,

(iii) for any $x \in l_{\Phi, p}, \varepsilon>0$ there exists $\delta>0$ such that, for every $y \in l_{\Phi, p} \backslash\{0\}$, there holds $\left\|y^{(1)}\right\|<\varepsilon / 3$, where $y=y^{(1)}+y^{(2)}, y^{(1)}=\Sigma_{j \in A} y(j) \xi_{j}, y^{(2)}=\Sigma_{j \notin A} y(j) \xi_{j}$, $\xi_{j}: \xi(j)=1, \xi(m)=0$ for any $m \neq j$, and

$$
\begin{aligned}
A & =\left\{j \in \mathbb{N}: \Sigma_{e= \pm 1, \pm i}|x(j)+e y(j)|\right. \\
& \leq 4(1+\delta)|x(j)|\} .
\end{aligned}
$$

We will show that the Orlicz modular sequence space $l_{\Phi, \rho}$ is complex midpoint locally uniformly convex without any condition.

Theorem 15. Let $l_{\Phi, \rho}$ be an Orlicz modular sequence space. Then $l_{\Phi, \rho}$ is complex midpoint locally uniformly convex.

Proof. Suppose that $x \in S\left(l_{\Phi, \rho}\right)$ is not a complex strongly extreme point of the unit ball $B\left(l_{\Phi, \rho}\right)$. By Definition 4 , then there exists $\varepsilon_{0}>0$ such that $\Delta_{c, \rho}\left(x, \varepsilon_{0}\right)=0$. In a similar way to Proposition 8 , we can find a sequence $\left\{z_{n}\right\} \subseteq l_{\Phi, \rho}$ satisfying $\rho\left(z_{n}\right) \geq \varepsilon_{0}$ and

$$
\sup _{|\lambda| \leq 1} \rho_{\Phi}\left(x+\lambda z_{n}\right) \leq \frac{1}{\left|\lambda_{n}\right|}
$$

For the above $\varepsilon_{0}>0$, by Lemma 11, there exists $\delta_{0} \epsilon$ $(0,1 / 2)$, such that if $u, v \in \mathbb{C}$ and

$$
|v| \geq \frac{\varepsilon_{0}}{8} \max _{e}|u+e v|,
$$

then

$$
|u| \leq \frac{1-2 \delta_{0}}{4} \Sigma_{e}|u+e v|
$$

For every $n \in \mathbb{N}$, let

$$
\begin{aligned}
& A_{n}=\left\{j \in \mathbb{N}:\left|z_{n}(j)\right| \geq \frac{\varepsilon_{0}}{8} \max _{e}\left|x(j)+e z_{n}(j)\right|\right\}, \\
& z_{n}^{(1)}: z_{n}^{(1)}(j)=z_{n}(j) \quad\left(j \notin A_{n}\right), \\
& z_{n}^{(1)}(j)=0 \quad\left(j \in A_{n}\right), \\
& z_{n}^{(2)}: z_{n}^{(2)}(j)=0 \quad\left(j \notin A_{n}\right), \\
& z_{n}^{(2)}(j)=z_{n}(j) \quad\left(j \in A_{n}\right) .
\end{aligned}
$$

It is easy to see that $z_{n}=z_{n}^{(1)}+z_{n}^{(2)}$ for every $n \in \mathbb{N}$, and

$$
\begin{aligned}
\rho_{\Phi}\left(z_{n}^{(1)}\right) & =\sum_{j \notin A_{n}} \Phi\left(\left|z_{n}(j)\right|\right) \\
& \leq \sum_{j \notin A_{n}} \Phi\left(\frac{\varepsilon_{0}}{8} \max _{e}\left|x(j)+e z_{n}(j)\right|\right) \\
& \leq \frac{\varepsilon_{0}}{8} \sum_{j \notin A_{n}} \Phi\left(\max _{e}\left|x(j)+e z_{n}(j)\right|\right) \\
& \leq \frac{\varepsilon_{0}}{8} \sum_{e} \rho_{\Phi}\left(x+e z_{n}\right) \leq \frac{\varepsilon_{0}}{2\left|\lambda_{n}\right|}<\frac{3 \varepsilon_{0}}{4}
\end{aligned}
$$

for $n$ large enough since $\left|\lambda_{n}\right| \rightarrow 1$ as $n \rightarrow \infty$. Consequently, we obtain

$$
\rho_{\Phi}\left(z_{n}^{(2)}\right)>\frac{\varepsilon_{0}}{4}
$$

which shows that $A_{n} \neq \emptyset$. Furthermore, we have

$$
\begin{aligned}
1= & \rho_{\Phi}(x)=\sum_{j \in A_{n}} \Phi(|x(j)|)+\sum_{j \notin A_{n}} \Phi(|x(j)|) \\
\leq & \sum_{j \in A_{n}} \Phi\left(\frac{1-2 \delta_{0}}{4} \Sigma_{e}\left|x(j)+e z_{n}(j)\right|\right) \\
& +\sum_{j \notin A_{n}} \Phi\left(\frac{1}{4} \Sigma_{e}\left|x(j)+e z_{n}(j)\right|\right)
\end{aligned}
$$




$$
\begin{aligned}
\leq & \left(1-2 \delta_{0}\right) \sum_{j \in A_{n}} \Phi\left(\frac{1}{4} \Sigma_{e}\left|x(j)+e z_{n}(j)\right|\right) \\
& +\sum_{j \notin A_{n}} \Phi\left(\frac{1}{4} \Sigma_{e}\left|x(j)+e z_{n}(j)\right|\right) \\
= & \sum_{j=1}^{\infty} \Phi\left(\frac{1}{4} \sum_{e}\left|x(j)+e z_{n}(j)\right|\right) \\
& -2 \delta_{0} \sum_{j \in A_{n}} \Phi\left(\frac{1}{4} \Sigma_{e}\left|x(j)+e z_{n}(j)\right|\right) \\
\leq & \frac{1}{4} \sum_{j=1}^{\infty} \Phi\left(\sum_{e}\left|x(j)+e z_{n}(j)\right|\right) \\
& -2 \delta_{0} \sum_{j \in A_{n}} \Phi\left(\frac{1}{4} \Sigma_{e}\left|x(j)+e z_{n}(j)\right|\right) \\
\leq & \frac{1}{\left|\lambda_{n}\right|}-2 \delta_{0} \sum_{j \in A_{n}} \Phi\left(\frac{1}{4} \Sigma_{e}\left|x(j)+e z_{n}(j)\right|\right) .
\end{aligned}
$$

Notice that

$$
\begin{aligned}
& \sum_{j \in A_{n}} \Phi\left(\frac{1}{4} \sum_{e}\left|x(j)+e z_{n}(j)\right|\right) \geq \sum_{j \in A_{n}} \Phi\left(\left|z_{n}(j)\right|\right) \\
& \quad=\rho_{\Phi}\left(z_{n}^{(2)}\right)>\frac{\varepsilon_{0}}{4} .
\end{aligned}
$$

Hence,

$$
\begin{aligned}
1 & =\rho_{\Phi}(x) \\
& \leq \frac{1}{\left|\lambda_{n}\right|}-2 \delta_{0} \sum_{j \in A_{n}} \Phi\left(\frac{1}{4} \Sigma_{e}\left|x(j)+e z_{n}(j)\right|\right) \\
& <\frac{1}{\left|\lambda_{n}\right|}-\frac{\delta_{0} \varepsilon_{0}}{2} .
\end{aligned}
$$

In view of $\left|\lambda_{n}\right| \rightarrow 1$ as $n \rightarrow \infty$, letting $n \rightarrow \infty$, then we get a contradiction

$$
1=\rho_{\Phi}(x)<\frac{1}{\left|\lambda_{n}\right|}-\frac{\delta_{0} \varepsilon_{0}}{2}<1,
$$

which completes the proof.

Combining Corollary 10 and Theorem 15 we obtain immediately the following result.

Corollary 16. Let $l_{\Phi, \rho}$ be an Orlicz modular sequence space. Then $l_{\Phi, \rho}$ is complex strictly convex.

\section{Competing Interests}

The authors declare that there is no conflict of interests regarding the publication of this paper.

\section{Acknowledgments}

This work was supported in part by the National Natural Science Foundation of China under Grant 11401141, Heilongjiang Provincial Natural Science Foundation for Youths under Grant QC2013C001, Heilongjiang Provincial Postdoctoral Science Foundation under Grant LBH-Z15098, and Natural Science Foundation of Heilongjiang Educational Committee under Grant 12531099.

\section{References}

[1] E. Thorp and R. Whitley, "The strong maximum modulus theorem for analytic functions into a Banach space," Proceedings of the American Mathematical Society, vol. 18, no. 4, pp. 640646, 1967.

[2] J. Globevnik, "On complex strict and uniform convexity," Proceedings of the American Mathematical Society, vol. 47, no. 1, pp. 175-178, 1975.

[3] W. Davis, D. Darling, and N. Tomczak-Jaegermann, "The complex convexity for quasi-normed linear spaces," Journal of Functional Analysis, vol. 55, no. 1, pp. 110-150, 1984.

[4] P. N. Dowling, Z. B. Hu, and D. Mupasiri, "Complex convexity in Lebesgue-Bochner function spaces," Transactions of the American Mathematical Society, vol. 348, no. 1, pp. 127-139, 1996.

[5] O. Blasco and M. Pavlović, "Complex convexity and vectorvalued Littlewood-Paley in-equalities," Bulletin of the London Mathematical Society, vol. 35, no. 6, pp. 749-758, 2003.

[6] C. Choi, A. Kamińska, and H. J. Lee, "Complex convexity of Orlicz-Lorentz spaces and its applications," Bulletin of the Polish Academy of Sciences. Mathematics, vol. 52, no. 1, pp. 19-38, 2004.

[7] H. Hudzik and A. Narloch, "Relationships between monotonicity and complex rotundity properties with some consequences," Mathematica Scandinavica, vol. 96, no. 2, pp. 289-306, 2005.

[8] H. J. Lee, "Monotonicity and complex convexity in Banach lattices," Journal of Mathematical Analysis and Applications, vol. 307, no. 1, pp. 86-101, 2005.

[9] H. J. Lee, "Complex convexity and monotonicity in quasiBanach lattices," Israel Journal of Mathematics, vol. 159, pp. 5791, 2007.

[10] M. M. Czerwińska and A. Kamińska, "Complex rotundities and midpoint local uniform rotundity in symmetric spaces of measurable operators," Studia Mathematica, vol. 201, no. 3, pp. 253-285, 2010.

[11] M. M. Czerwińska and A. Parrish, "Complex extreme points in Marcinkiewicz spaces," Positivity, vol. 19, no. 1, pp. 121-135, 2015.

[12] L. Chen, Y. Cui, and H. Hudzik, "Criteria for complex strongly extreme points of Musielak-Orlicz function spaces," Nonlinear Analysis, Theory, Methods and Applications, vol. 70, no. 6, pp. 2270-2276, 2009.

[13] S. Chen, Geometry of Orlicz Spaces, Dissertationes Mathematicae, Warsaw, Poland, 1996.

[14] Y. Cui, H. Hudzik, and R. Pluciennik, "Extreme points and strongly extreme points in Orlicz spaces equipped with the Orlicz norm," Zeitschrift Für Analysis und Ihre Anwendungen, vol. 22, no. 4, pp. 789-817, 2003.

[15] Y. Cui, L. Duan, H. Hudzik, and M. Wisła, "Basic theory of $p$ Amemiya norm in Orlicz spaces $(1 \leq p \leq \infty)$ : extreme points and rotundity in Orlicz spaces endowed with these norms," Nonlinear Analysis, Theory, Methods and Applications, vol. 69, no. 5-6, pp. 1796-1816, 2008. 
[16] Y. Cui, H. Hudzik, R. Kumar, and L. Maligranda, "Composition operators in Orlicz spaces," Journal of the Australian Mathematical Society, vol. 76, no. 2, pp. 189-206, 2004.

[17] Y. Cui, H. Hudzik, J. Li, and M. Wisła, "Strongly extreme points in Orlicz spaces equipped with the $p$-Amemiya norm," Nonlinear Analysis: Theory, Methods \& Applications, vol. 71, no. 12, pp. 6343-6364, 2009.

[18] P. Foralewski, H. Hudzik, and R. Płuciennik, "Orlicz spaces without extreme points," Journal of Mathematical Analysis and Applications, vol. 361, no. 2, pp. 506-519, 2010.

[19] A. Kamińska, "On uniform convexity of Orlicz spaces," Indagationes Mathematicae, vol. 44, no. 1, pp. 27-36, 1982.

[20] A. Kamińska, "On some convexity properties of MusielakOrlicz spaces," Rendiconti del Circolo Matematico di Palermo. Serie II. Supplemento ai Rendiconti del Circolo Matemàtico di Palermo, no. 5, pp. 63-72, 1984.

[21] L. Maligranda, Orlicz Spaces and Interpolation, University of Campinas, Campinas, Brazil, 1989.

[22] M. M. Rao and Z. D. Ren, Theory of Orlicz Spaces, vol. 146 of Monographs and Textbooks in Pure and Applied Mathematics, Marcel Dekker, New York, NY, USA, 1991.

[23] M. A. Khamsi, W. M. Kozlowski, and S. T. Chen, "Some geometrical properties and fixed point theorems in Orlicz spaces," Journal of Mathematical Analysis and Applications, vol. 155, no. 2, pp. 393-412, 1991.

[24] P. D. Liu and Y. Hou, "On the geometry of complex Banach spaces," Advances in Mathematics, vol. 27, no. 1, pp. 1-20, 1998.

[25] M. A. Khamsi, W. M. Kozlowski, and S. Reich, "Fixed point theory in modular function spaces," Nonlinear Analysis: Theory, Methods \& Applications, vol. 14, no. 11, pp. 935-953, 1990.

[26] W. M. Kozlowski, Modular Function Spaces, Marcel Dekker, New York, NY, USA, 1988.

[27] H. Hudzik, "Monotonicity and best approximation in Banach lattices," Acta Mathematica Sinica, vol. 25, no. 5, pp. 785-794, 2009.

[28] H. Hudzik, W. Kowalkwski, and M. Wis la, "Approximative compactness and continuity of metric projector in Banach spaces and applications," Science China Mathematics, vol. 51, no. 2, pp. 293-303, 2008.

[29] J. Musielak, Orlicz Spaces and Modular Spaces, Springer, Berlin, Germany, 1983.

[30] J. Musielak and W. Orlicz, “On modular spaces," Studia Mathematica, vol. 18, pp. 49-65, 1959.

[31] J. Musielak and W. Orlicz, "Some remarks on modular spaces," Bulletin of the Polish Academy of Sciences: Mathematics, vol. 7, pp. 661-668, 1959.

[32] H. Nakano, Modular Semi-Ordered Linear Spaces, Maruzen, Tokyo, Japan, 1950.

[33] W. Orlicz, "A note on modular spaces," Bulletin of the Polish Academy of Sciences: Mathematics, vol. 9, pp. 157-162, 1961.

[34] L. Chen and Y. Cui, "Complex rotundity of Orlicz sequence spaces equipped with the $p$-Amemiya norm," Journal of Mathematical Analysis and Applications, vol. 378, no. 1, pp. 151-158, 2011. 


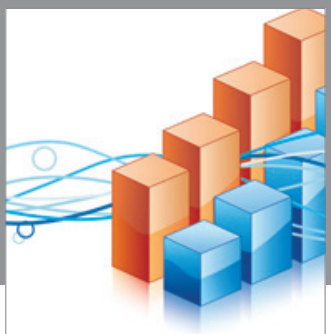

Advances in

Operations Research

vatem alat4

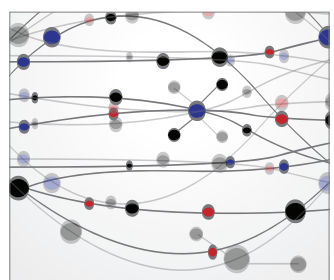

\section{The Scientific} World Journal
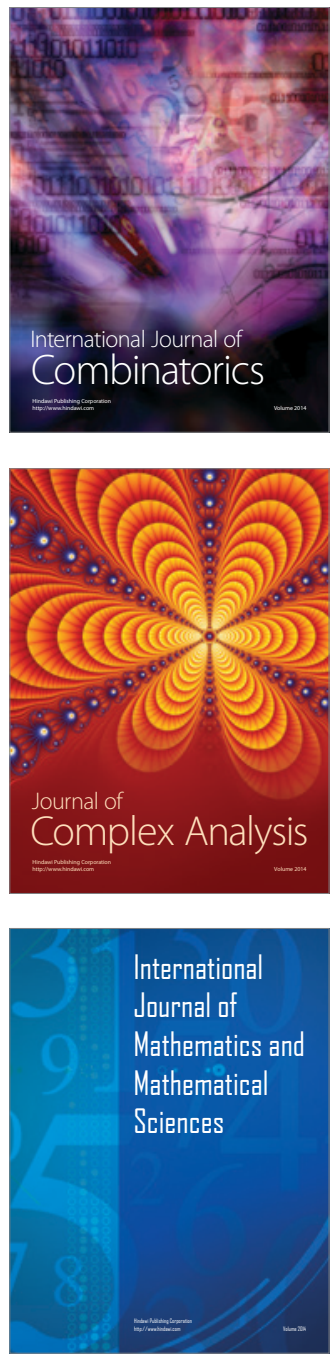
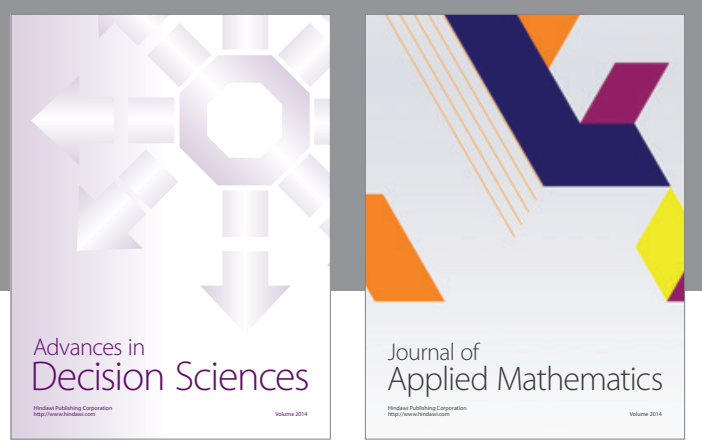

Algebra

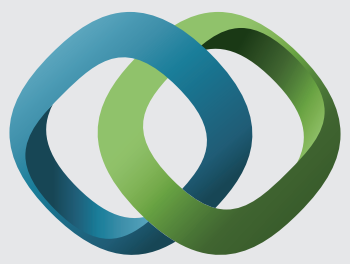

\section{Hindawi}

Submit your manuscripts at

http://www.hindawi.com
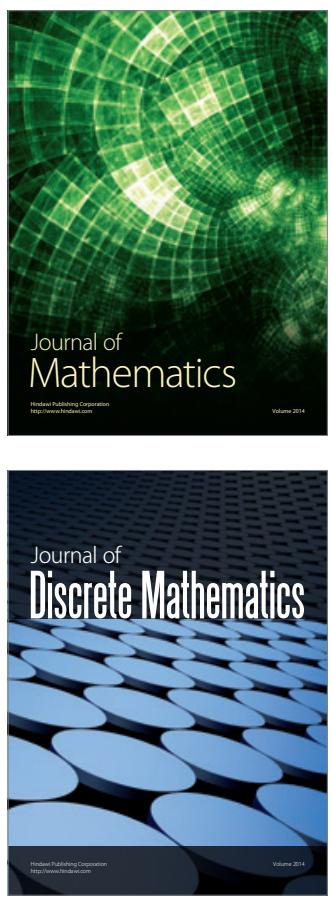

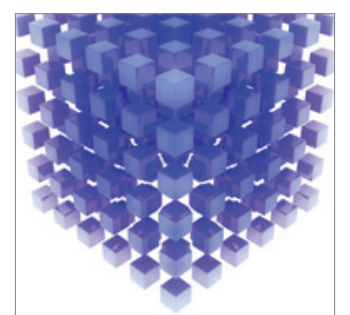

Mathematical Problems in Engineering
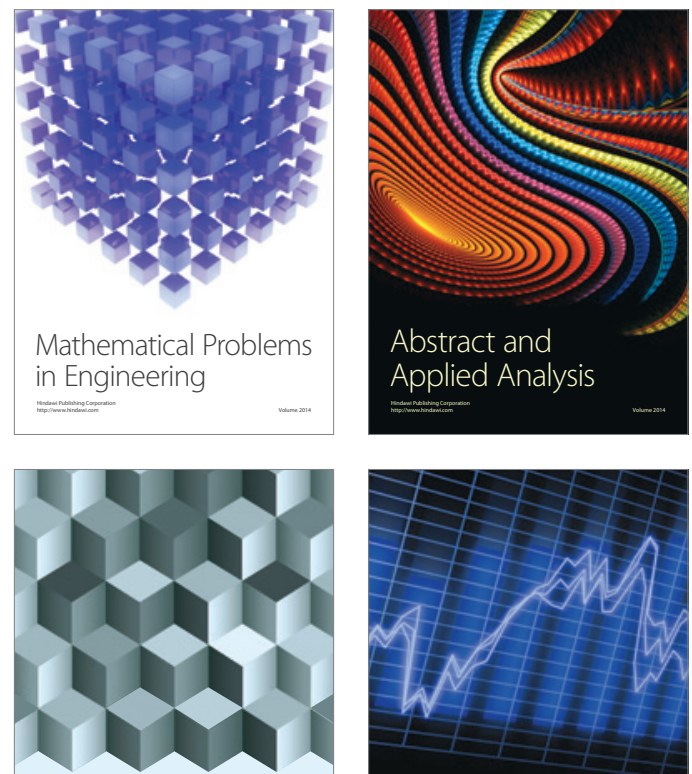

Journal of

Function Spaces

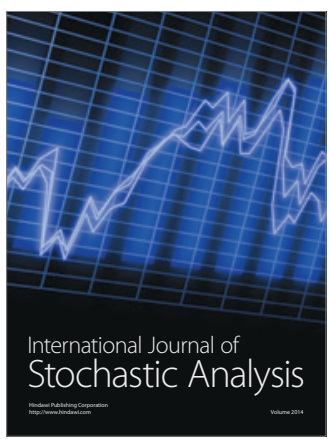

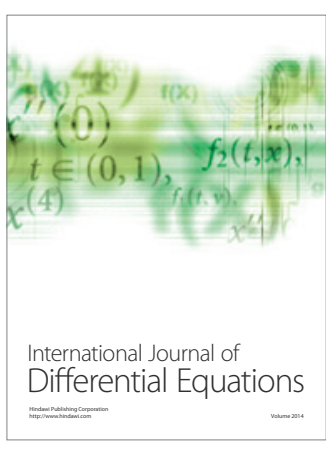
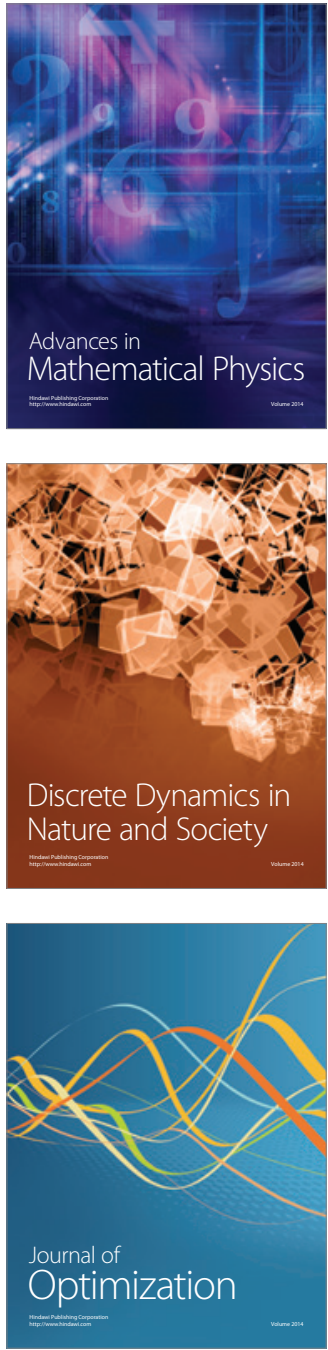